\section{Biochemical Therapeutic Benefits of Garlic on Atherosclerosis Induced by Soybean in Rats}

\section{Abstract}

Setting: For centuries, garlic (Allium sativum. L) plays an important medicinal role and culinary value. Cardiovascular disease (CV) is a complex disease associated with hypercholesterolemia.

Aim of the work: The study was designed to evaluate the efficacy of dietary supplementation with garlic juice on hypercholesterolemia induced by a high dietary soybean oil extract and cholesterol in animal (male wistar rats).

Animals and methods: Animals were divided into three groups (each group was eight rats). Group one (control rats group) was fed with a control diet made up of the normal rat chow (C), group two of animals was fed with a hypercholesterolemic diet (HPC) enriched with $25 \%$ soybean oil and $1 \%$ cholesterol for 8 weeks and group three received a hypercholesterolemic diet (HPC) enriched with $25 \%$ soybean oil and $1 \%$ cholesterol as in second group simultaneously with daily oral administration of $1 \mathrm{ml}$ of garlic juice/100 g body weight (equivalent to 4/10 g/100 g BW) for 8 weeks. Results: The total cholesterol concentrations (TC), the low density lipoprotein concentrations (LDL-C), the high density lipoprotein concentrations (HDL-C), and the ratio of triglycerides were investigated. The concentrations of total cholesterol (TC), low density lipoprotein (LDL-C) and the triglycerides was significantly increased in the serum of rats fed with the dietary intake of the hypercholesterolemic (HPC) diet. No significant difference in the HDL-C concentrations. Rats which were fed with the hypercholesterolemic (HPC) diet was observed weight loss of body and organs, heart, kidney, liver and abdominal fat. Significant better lipidemic status and weight improvement of the body and organs showed with the rats on a hypercholesterolemic (HPC) diet treated with a garlic juice. Therefore, the study suggested that garlic juice is an important determinant of serum lipid concentrations and weight, so it is considered as an antilipidaemic agent against the pathogenesis of atherosclerosis.

Keywords: Experimental animals; Hypercholesterolemia; Biochemical tests; Atherosclerosis; Garlic juice

\section{El-Khedr Mohamed Mostafa El-Gamal* and Usama Bhgat Mostafa El- Gazzar}

Faculty of Medicine (Dommitta), Department of Biochemistry, Al-Azher University, Cairo, Egypt

\section{*Corresponding author:}

El-Khedr Mohamed Mostafa El-Gamal

\section{झ khedr_bio@yahoo.com}

Faculty of Medicine (Dommitta), Department of Biochemistry, Al-Azher University, Cairo, Egypt.

Tel: 00201098511073

Citation: El- Gamal EMM,

El-Gazzar UBM (2017) Biochemical

Therapeutic Benefits of Garlic on

Atherosclerosis Induced by Soybean in Rats.

Biochem Mol Biol J. Vol.3 No.3:19

Received: October 30, 2017; Accepted: December 05, 2017; Published: December 11,2017

\section{Introduction}

Cholesterol increasing concentration (Hypercholesterolemia) is a lipoprotein metabolic disorder characterized by high serum concentration of low density lipoprotein (LDL-C) and blood serum cholesterol [1]. Atherosclerosis can be prevented by decreasing risk factors through: healthy eating, exercise, avoidance of tobacco smoke and limiting alcohol intake. Hypercholesterolemia poses a major problem to many societies as well as health pro- fessionals because of relative higher mortality from Ischemic Heart Disease (IHD). Elevation of the total cholesterol (TC) and low-density lipoprotein (LDL) are well established risk factors of atherogenesis [2]. The earliest lesion of atherosclerosis is the simple fatty streak, which eventually changes to fibrous plaques resulting in arterial occlusion, thereby producing overt clinical manifestations [3]. A number of clinical investigations, of oral antilipidaemic agents from plants extractions used in traditional medicine, have been investigated and many of the plants were 
found with high activity [4]. Garlic (Allium sativum Linn) has been used by man in different cultures for hundreds of years as foodstuffs, condiments, as flavourings and in folk medicine. The World Health Organization (WHO) has also recommended the evaluation of the plants' effectiveness in conditions where we lack safe and modern drugs [5]. This has leaded to an increasing demand of research on natural antilipidemic plants which produces minimal or no side effects to cardiovascular disease. Garlic plant (A)lium sativum L.) is a common spicy flavouring agent used since ancient times. Some protective effects of garlic have been well established by epidemiological studies and animal experiments in the past decade. Investigation of the commercially available garlic preparations in the form of garlic oil, garlic powder, and pills which are widely used for certain therapeutic purposes, including lowering blood pressure and improving lipid profile leading to improvement of cardiovascular and other disease were performed by Elkayam, Mirelman and Peleg [6]. Garlic has been largely attributed to the reduction of risk factors for cardiovascular diseases and cancer [7], activaton of immune function [8], protection of liver [9] and has antioxidant effect [10]. At least, garlic extract contains 33 sulfur compounds, several enzymes, 17 amino acids, and minerals such as selenium. Also, garlic plant has numerous biological activities that are attributed to its rich content of different volatile organosulfur compound (OSC) and other phytochemicals that work in synergically by combination of mechanisms for substance acting on various molecular targets [11-14]. Garlic or extracts has hundreds of chemical compounds and they were estimated biochemically. Some basic sulphur-containing bioactive constituents in whole, intact garlic are the 8-glutamyl S-alk(en)yl-L-cysteines and S-lk(en)yl-L-cysteine sulphoxides, including alliin substance. The 8 -glutamyl peptides are biosynthetic intermediates for corresponding cysteine sulphoxides compound $[15,16]$. Whole intact garlic typically contains $\sim 1 \%$ alliin compound, together with (+)S -methyl- L-cysteine sulphoxide (methiin) substance and (+)S -(trans-1-propenyl)-L-cysteine sulphoxide compound. S-(2-carboxypropyl) glutathione, 8-glutamyl S -allyl-Lcysteine, 8-glutamyl S -(trans-1-pro- pynyl)-L-cysteine and 8 -glutamyl S-allyl mercapto-L-cysteine are also reported to be present in garlic cloves parts $[16,17]$. The major health benefits of garlic likely arise from a wide variety of components, possibly working synergistically. Moreover, it is proposed that the prediction of potential health benefit(s) from garlic plant is largely dependent on the efficiency and safety of the garlic preparations, which are also containing on the processing methods employed. The complex chemistry structures of garlic extract makes it plausible that variations in processing can yield quite different preparations. Highly unstable thiosulphinates compounds, such as allicin, dis- appear during processing method and are quickly transformed into a variety of organo-sulphur components. In spite of, there are many garlic supplements commercially available now, they fall into one of four categories, which are includes 1- dehydrated garlic powder, 2-garlic oil, 3- garlic oil macerate and 4- aged garlic extract ( $A G E$ ). Garlic has been appraised as a remedy for the cure and prevention of a number of diseases such as cardiovascular and cerebrovascular diseases, as well as other metabolic diseases, high lipid profile (hyperlipidaemia) and diabetes mellitus [18]. The purpose of the present study is firstly to evaluate the influence of oral administration of garlic juice on Hypercholesterolemia induced by a high dietary soybean oil and cholesterol in rats.

\section{Materials and Methods}

\section{Method of garlic juice preparation}

From the local market in New Damietta, Egypt, bulbs of fresh garlic (Allium sativum Linn) were purchased., peeled, washed, and chopped into small pieces. One hundred gram of chopped garlic were added and crushed in a mixing machine with $250 \mathrm{ml}$ of distilled water. Slurry produced was squeezed and filtered through a fine cloth and the filtrate was quickly frozen at $-10^{\circ} \mathrm{C}$ until used [19].

\section{Rats and treatments}

From the animal house at the faculty of Medicine (Damietta), University of Al-Azhar, Egypt, twenty four male weanling rats weighing approximately (60-80 g) were obtained. Rats were housed in three groups (each group was 8 rats) in a controlled environment with 12 hour light and 12 hour dark cycles. They were allowed free access to different dietary formulations (Table 1) and water ad libitum for 8 weeks. The first group of animals was fed with the control diet (C), the second group of animals was fed with a hypercholesterolemic diet (HPC) [20] and finally, the third group of rats was fed with a hypercholesterolemic diet (HPC) with simultaneous administration of $1 \mathrm{ml}$ of garlic juice daily oral by gavage/100 g body weight (equivalent to $4 / 10 \mathrm{~g} / 100 \mathrm{~g}$ BW) [21]. Every week, the rats were weighed throughout the period of the experiment. Proximate analysis of both the control and hypercholesterolemic diets of the rats were determined (Table 2) using the methods described by AOAC [22] for crude protein, crude fat, crude fibre, and ash while carbohydrate was determined by subtracting the sum of the other nutrient parameters from 100.

\section{Assay kits and reagents}

The assay kits for cholesterol, HDL-C, LDL-C, triglycerides, were obtained from Randox Laboratories Ltd., Ardmore, Co. Antrim, UK. All remainder reagents used were of analytical grade. The

Table 1 Control diet composition, hypercholesterolemic diet composition and hypercholesterolemic diet composition $+1 \mathrm{ml} \mathrm{Garlic} \mathrm{juice} \mathrm{fed} \mathrm{to} \mathrm{the}$ male rats.

\begin{tabular}{|c|c|c|c|c|c|c|}
\hline Ingredient g/kg rats & Mineral/Vitamin mix & Soybean oil & Cellulose & Cholesterol & Casein & Corn starch \\
\hline Control diet group & 90.0 & 90.0 & 30.0 & 0.0 & 140.0 & 839.8 \\
\hline HPC diet group & 90.0 & 340.0 & 230.0 & 30.0 & 140.0 & 539.8 \\
\hline HPC diet + 1 ml garlic juice & 90.0 & 340.0 & 230.0 & 30.0 & 140.0 \\
\hline
\end{tabular}


animals were fasted overnight at the end of the experiment (after eight weeks), sacrificed under ether anaesthesia and blood collected from the jugular vein for analysis. Centrifugation of the blood samples were performed at $1200 \times \mathrm{g}$ for $5 \mathrm{~min}$ and the serum was collected in Eppendorf tubes for analysis. The heart, liver and kidney of each animal was removed, weighed and stored at $-80^{\circ} \mathrm{C}$; carefully dissection of abdominal fat and weighed was also made. The relative organ to body weight was calculated. The concentration of total cholesterol (TC), HDL-C and triglycerides were determined in the serum of the rats by adopting the protocol outlined in the manufacturer's assay kit from Randox Laboratories Ltd, Ardmore, Co. Antrim, UK. The concentration of LDL-C was calculated using the Friedewald formula, LDL-C $=T C$ (HDL-C+TG/5).

\section{Statistical analysis}

Data experiment of all result were expressed as mean \pm Standerd Deviation (S.D) and were statistically analysed using one way analysis of variance (ANOVA). The data of the means were separated by the Duncan multiple test using SAS [23]. All values of results data were considered significant at $(p<0.05)$ and very significant at $(p<0.01)$.

\section{Results}

After eight weeks of a hypercholesterolemic diet feeding, there were a significant difference in the weight loss pattern of the rats fed with hypercholesterolemic (HPC) diet compared to those fed with the control diet. While, significant increase in weight was achieved in garlic-treated group (Table 3 ). Concentration of the serum lipid of the cholesterol fed rats increased many folds to that of the initial concentration except in the normal control group (Table 4). The serum cholesterol profile increased maximally to almost 5.4 times the initial concentration and observed markedly higher level of lipid profiles concentration. Elevation of the serum triglycerides concentration was relatively lower as compared to serum low-density lipoprotein (LDL) and cholesterol level. The mean final serum high-density lipoprotein (HDL) con-

Table 2 Proximate composition of the control diet, hypercholesterolemic diet and hypercholesterolemic diet $+1 \mathrm{ml}$ garlic juice diets fed to the male Wistar rats.

\begin{tabular}{|c|c|c|c|}
\hline Parameter (\%) & Control diet group & HPC diet group & HPC diet group +1 ml garlic juice \\
\hline Ash & $9.24 \pm 0.16$ & $6.73 \pm 0.04$ & $6.73 \pm 0.04$ \\
\hline Crude protein & $26.95 \pm 0.14$ & $28.74 \pm 0.02$ & $28.74 \pm 0.02$ \\
\hline Crude fat & $19.64 \pm 0.04$ & $33.44 \pm 0.14$ & $33.44 \pm 0.14$ \\
\hline Moisture & $7.90 \pm 0.14$ & $8.15 \pm 0.07$ & $8.15 \pm 0.07$ \\
\hline Crude fibre & $2.99 \pm 0.14$ & $3.25 \pm 0.14$ & $3.25 \pm 0.14$ \\
\hline Total carbohydrate & $39.25 \pm 0.13$ & $37.27 \pm 0.16$ & $37.25 \pm 0.13$ \\
\hline
\end{tabular}

Table 3 Weight body and organs with different diets of male Wistar rats.

\begin{tabular}{|c|c|c|c|}
\hline Organ (g) & Control rats & HPC rats & HPC rats + 1 ml garlic juice \\
\hline Kidney & $3.00 \pm 0.00$ & $3.10 \pm 0.00$ & 3. $00 \pm 0.00$ \\
\hline liver & $10.00 \pm 1.41$ & $8.18 \pm 0.25$ & $9.60 \pm 1.41 *$ \\
\hline Heart & $4.53 \pm 0.18$ & 3. $29 \pm 0.09$ & $3.93 \pm 0.18$ \\
\hline Abdominal fat & $7.27 \pm 0.69$ & $8.40 \pm 0.08$ & $5.97 \pm 0.69 *$ \\
\hline Body weight & $156.84 \pm 4.01$ & $112.50 \pm 12.50$ & $149.65 \pm 6.4^{*}$ \\
\hline
\end{tabular}

Table 4 Concentration of serum lipid in all groups of rats at the starting and ending of the trial.

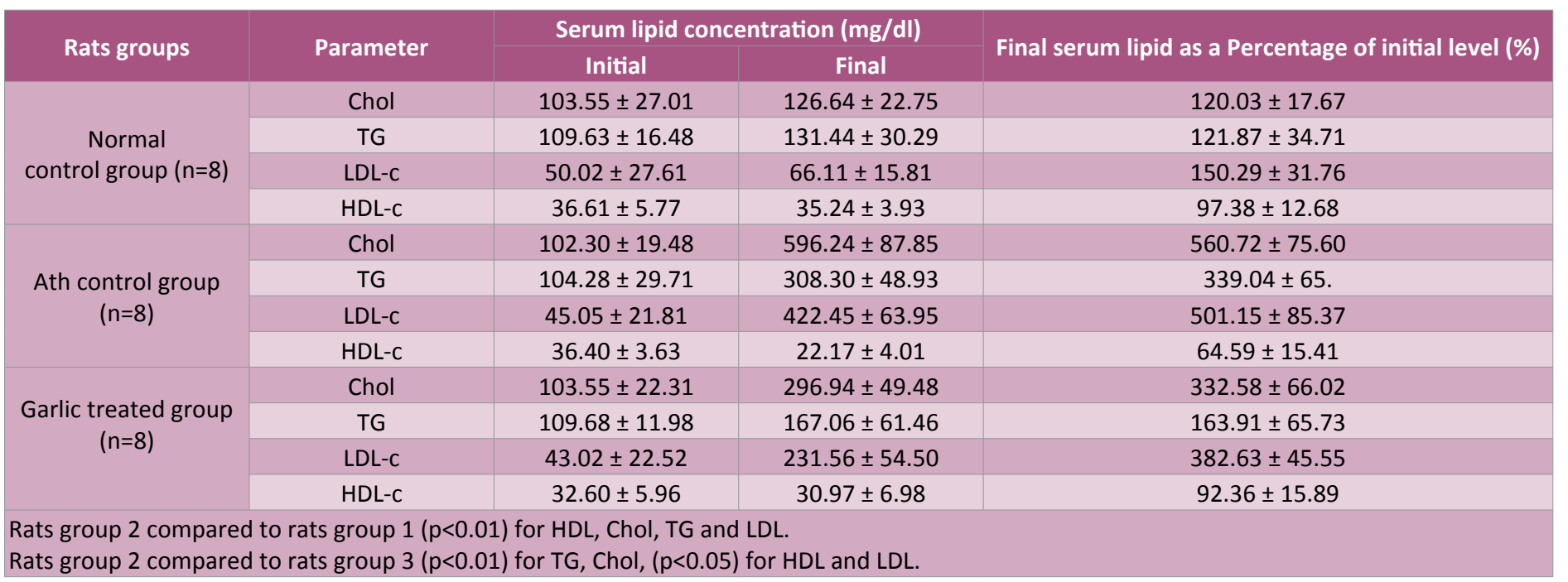


centration were less than the mean initial ones in all groups of rats. Levels of the mean final serum cholesterol concentration in the a hypercholesterolemic control group was 5.4 times more than that in the normal control ones. The mean of the final serum cholesterol concentration as percentage of the initial ones in the normal control rats was $120.02 \pm 17.08 \mathrm{mg} / \mathrm{dl}$ and that of the hypercholesterolemic control ones was $560.73 \pm 85.60 \mathrm{mg} /$ dl. Statistically, very significantly $(p<0.01)$ higher difference was found when the two groups of rats were compared for the final serum cholesterol concentration expressed as a percentage of the corresponding initial concentration. The mean final serum cholesterol level in the garlic treated rats was $296.92 \pm 49.49$ $\mathrm{mg} / \mathrm{dl}$, which was almost 2.7 times higher than that of the corresponding mean initial level $(103.55 \pm 22.31 \mathrm{mg} / \mathrm{dl})$. Also, the mean of the final concentration expressed as percentages of the corresponding initial concentration $(332.58 \pm 66.05 \mathrm{mg} / \mathrm{dl})$ was very significantly lower $(p<0.01)$ than that in the hypercholesterolemic control ones $(560.72 \pm 75.60 \mathrm{mg} / \mathrm{dl})$. Results showed that, the mean final of serum triglycerides concentration and that was expressed as percentage of the corresponding initial levels in the normal control group was $131.44 \pm 30.33 \mathrm{mg} / \mathrm{dl}$ and 121.87 $\pm 34.72 \mathrm{mg} / \mathrm{dl}$ respectively. The mean final serum triglycerides concentration in the hypercholesterolemic control group was $308.30 \pm 48.88 \mathrm{mg} / \mathrm{dl}$, almost double than that of the mean final value in the normal control group. From the Table 4, the mean of the final triglycerides concentration expressed as percentages of the corresponding initial ones in this group $(329.05 \pm 95.50 \mathrm{mg} /$ dl) was very significantly higher $(p<0.01)$ than that in the normal control ones. Mean final level of serum triglycerides concentration in the garlic treated rats was $167.06 \pm 61.53 \mathrm{mg} / \mathrm{dl}$ and the mean of the final concentrations expressed as percentages of the corresponding initial levels $(163.91 \pm 65.80 \mathrm{mg} / \mathrm{dl})$ was very significantly lower $(p<0.01)$ than that in the hypercholesterolemic control ones $(339.05 \pm 65.48 \mathrm{mg} / \mathrm{dl})$. At the same time, the mean final serum concentration and the levels expressed as percentages of the corresponding initial levels in normal control group of low density-lipoprotein (LDL) was $66.14 \pm 15.80 \mathrm{mg} / \mathrm{dl}$ and 150.28 $\pm 31.75 \mathrm{mg} / \mathrm{dl}$ respectively. The mean final serum of low densitylipoprotein (LDL) concentration in the hypercholesterolemic control group $(422.46 \pm 63.88 \mathrm{mg} / \mathrm{dl})$ was almost 6 times than that of the mean final low-density lipoprotein (LDL) concentration-in the normal control ones $(66.12 \pm 25.82 \mathrm{mg} / \mathrm{dl})$. Statistically, the mean of the final serum of low density-lipoprotein (LDL) concentration expressed as percentages of the corresponding initial levels in this group $(501.15 \pm 85.28 \mathrm{mg} / \mathrm{dl})$ was very significantly higher $(p<0.01)$ than that in the normal control group $(150.29$ $\pm 31.76 \mathrm{mg} / \mathrm{dl}$ ). In the garlic treated rats group, the mean final low density-lipoprotein (LDL) concentration was $231.55 \pm 54.48$ $\mathrm{mg} / \mathrm{dl}$ and the mean of the final serum low density-lipoprotein (LDL) concentration expressed as percentages of the corresponding initial levels $(382.64 \pm 45.48 \mathrm{mg} / \mathrm{dl}$ ) was significantly lower $(p<0.05)$ than in the hypercholesterolemic control ones $(501.16 \pm$ $85.35 \mathrm{mg} / \mathrm{dl}$ ). On the other hand, the mean initial and final serum of high density-lipoprotein (HDL) concentration in the normal control group were $36.61 \pm 5.77 \mathrm{mg} / \mathrm{dl}$ and $35.24 \pm 3.88 \mathrm{mg} / \mathrm{dl}$ respectively. The mean final high density-lipoprotein (HDL) con- centration in the a hypercholesterolemic control group $(22.18 \pm$ $4.12 \mathrm{mg} / \mathrm{dl}$ ) was almost $30 \%$ lower than that of the mean final high density-lipoprotein (HDL) concentration in the normal control ones $(35.25 \pm 3.95 \mathrm{mg} / \mathrm{dl})$. The mean of the final serum high density-lipoprotein (HDL) concentrations expressed as percentages of the corresponding initial levels in this group (64.58 \pm 16 . $44 \mathrm{mg} / \mathrm{dl})$ was very significantly $(p<0.01)$ lower than that of the normal control group $(97.39 \pm 12.69 \mathrm{mg} / \mathrm{dl})$. On the other side, in the garlic treated rats fed on hypercholesterolemic diet the mean final high density-lipoprotein (HDL) concentration (29.95 \pm $6.88 \mathrm{mg} / \mathrm{dl}$ ) and that of the mean of the final serum high densitylipoprotein (HDL) concentrations expressed as percentages of the corresponding initial levels in this group $(92.35 \pm 15.88 \mathrm{mg} /$ $d l)$ was significantly higher $(p<0.05)$ than that in the a hypercholesterolemic control ones $(64.58 \pm 15.40 \mathrm{mg} / \mathrm{dl})$. Comparison of the final serum lipid (as percentage of the corresponding initial level) between different groups of rats were carried out through unpaired ' $t$ ' test of significance of difference.

\section{Discussion}

The effect of a hypercholesterolemic diet when compared to the outcome of the basal laboratory diet feeding could be assessed by comparing the findings in the a hypercholesterolemic control rats with those in the normal control ones. Highly significant difference between the two groups of animals could be detected statistically in all biochemical parameters measured during the experiment. Statistically, the mean final serum cholesterol concentration as well as the mean of the final levels expressed as percentages of the corresponding initial concentrations in the hypercholesterolemic control rats increased by about 5.4 times than that in the normal control ones. Matos et al. [20] and Harnafi et al. [24] confirms the same results in rats. Ahmed et al. [2] reported almost 8 times higher level of serum cholesterol concentration in the hypercholesterolemic control group than that in the normal control ones by feeding $6 \%$ coconut oil $+1 \%$ cholesterol to male albino rabbits for 10 weeks. In the garlic treated rats, the mean final cholesterol concentration was decreased by $60 \%$ than that in the hypercholesterolemic controls ones. Hartvigsen et al. Mohammadi and Oshaghi in the cholesterol [2] fed rats treated with garlic for eight weeks have reported similar decrement $[25,26]$. Reduction by $10 \%$ of serum cholesterol concentration after 3 hours in a healthy individual by simultaneous feeding of $50 \mathrm{gm}$ garlic juice with 100 butter and 4 pieces of bread have been spotted by Bordia et al. [27]. Reduction of serum cholesterol concentration has also been communicated by Haque [28] by intragastric administration of garlic in cholesterol fed rats for 10 weeks. Zahid et al. announce that garlic extract is a vasorelaxant and may decrease the atherogenic potentials of cholesterol concentration in rats [29]. Aged garlic extract ( $A G E$ ) has been acquaint to prevent lipid oxidation and oxidative modification of low density lipoprotein (LDL), just like that decreasing the amount of oxidized low density lipoprotein (LDL) in circulation and the subsequent build-up of cholesterol in macrophages, smooth muscles and blood vessel walls, leading to the suppression of atherogenic fatty streaks [30]. Both human and animal studies, the water and lipid soluble ingredient 
in garlic demoralize cholesterol synthesis [31]. Regarding to the cholesterol-lowering property of garlic, it has been suggested that some components of garlic may act as inhibitors for some enzymes such as hydroxy methyl glutaryl-CoA reductase, which collaborate in cholesterol biosynthesis. Consistent with this idea, it has been shown that in vivo treatment of garlic extract diminishes the lipid peroxidation products $[19,32]$. Final serum triglycerides concentration and the mean of the final levels expressed as percentages of the conformable foremost concentrations were more than 2.4 times and about $64 \%$ respectively, higher in the atherosclerotic control rats than that in the normal control ones. Similar higher concentrations have also been denounced by Getz and Reardon [33]. While, the mean final serum triglycerides concentration in the garlic treated rats was minimized by $45 \%$ than that in the atherosclerotic control rats. Jang and Wang also confirmed similar results [34]. More than 24\% depression has been manifested by Zacharias et al. in sucrose fed hypercholesterolemic rabbits treated with $10 \mathrm{ml} / \mathrm{kg}$ of aqueous extract of garlic for eight weeks [35]. Cicletane, [2] allicin, ajone and other sulphur-containing compounds in garlic may be responsible for the preservation of endothelial cell perfection via the prohibition of lipid peroxidative prejudice and depression in serum cholesterol and other oxidizable lipids [32]. Levels of the mean final serum low density lipoprotein (LDL) concentration in the atherosclerotic control rats increased by roughly 6 times than that in the normal controls ones. Ebrahimi et al. deliberated on the male rats found higher low-density lipoprotein (LDL) concentration (LDL) [36]. Ratios of the mean final serum low density lipoprotein (LDL) in the garlic treated rats was roughly $58 \%$ lower than that in the atherosclerotic control ones. Ebrahimi et al. have spotted about $50 \%$ depression of serum low density lipoprotein (LDL) concentration in a similar study. About $40 \%$ drooping in the low density lipoprotein (LDL) fed rats treated with aqueous extract of garlic for ten weeks was found by Haque [28]. The components, diallyl disulfide and diallyl trisulfide have been advertise by Lei et al. [13] to repress oxidized low-density lipoprotein (LDL)-induced vascular cell adhesion. Reports on the cardiovascular benefits of other garlic bioactive ingredients show that allicin had positively affect two atherosclerotic risk factors prohibiting the uptake of

\section{References}

1 Rerkasem K, Gallagher PJ, Grimble RF, Calder PC, Shearman CP (2014) Managing hypercholesterolemia and its correlation with carotid plaque morphology in patients undergoing carotid endoterectomy. Vascular Health Risk Manage 4: 1259-1264.

2 Ahmed AU, Begum SF, Islam HN, Naher BS (2007) The effect of garlic on cholesterol induced hyperlipidaemia in rabbits. Ibrahim Med Coll J 1: $16-20$

3 Clair RW (1983) Atherosclerosis regression in animal model: Current concepts of cellular and biochemical mechanism. Prog Cardiovascular Dis 26: 109-128.

4 Ugwu CE, Suru SM (2016) Medicinal use and health benefits of Allium sativum: A comparative review of the whole extract vs bioactive constituents. In: Haynes A, Allium sativum chemical constituents, medicinal uses and health benefits, Nova Science Publishers, New York, USA. pp. 43-69. low density lipoprotein (LDL) and breaking down of macrophages [37]. Also, the mean final serum of high density lipoprotein (HDL) concentration in the atherosclerotic control rats was about $30 \%$ lower than that in the normal control ones. [1] Same reduction was reported by Ugwu and Suru [4]. In the present study, the mean final serum high density lipoprotein (HDL) concentration in the garlic treated rats was inculcated by almost $25 \%$ than that in the atherosclerotic control ones. Comparable results were also communicated by Ali et al. [38] and Santhosha et al. [14]. They observed quite higher concentrations of serum high density lipoprotein (HDL) in the cholesterol fed rats treated with garlic. Haque found serum high density lipoprotein (HDL) concentration increased by $30 \%$ in the garlic treated rats in comparison to rats fed on cholesterol diet only. Garlic juice could directly ameliorate atherosclerosis by its capability to suppress arterial cell lipid content, suppress intracellular lipid accumulation as well as the inhibition of lipogenic and cholesterogenic hepatic enzymes activities [39]. Significant differences $(p<0.05)$ in the weight loss pattern of the rats fed with a hypercholesterolemic diet (HPC) contrasted to those fed with the control diet was spotted. While, significant $(p<0.05)$ acquisition in the weight was accomplished in garlic-treated group (Table 3). Losing of the weight assured by the organ to body weight ratio could be as a result of allowance in nutrient ingestion because of the high fat content of the diet which might have impaired the absorption of protein and other nutrients [20].

\section{Conclusion}

Rats on atherogenic diet treated with garlic showed better lipidemic status. On the other side, the untreated rats on a hypercholesterolemia diet showed significantly worst lipidemic status than that of the normal control ones as evident in higher serum triglycerides, cholesterol and low density lipoprotein (LDL), with lower serum high density lipoprotein (HDL) level, which apparent from significant difference between the two group. The present study has demonstrated that garlic has preventive effective against one of the deadliest killer disease (pathogenesis of atherosclerosis) due to the antilipidaemic role.

5 Mendis S, Puska P, Norrving B (2011) World health organization global atlas on cardiovascular disease prevention and control. World Health Organization in Collaboration with the World Heart Federation and the World Stroke Organization, Geneva, Switzerland. pp. 3-18.

6 Elkayam A, Mirelman D, Peleg E (2003) The effects of allicin on weight in fructose-induced hyperinsulinemic, hyperlipidemic, hypertensive rats. Am J Hypertension 16:1053-1056.

7 Thomson M, Al-Qattan KK, Bordia T, Muslim A (2006) Supplement: Significance of garlic and its constituents in cancer and cardiovascular disease. Including garlic in the diet may help lower blood glucose, cholesterol, and triglycerides. J Nutr 136: 800-802.

8 Salman H, Bergman M, Bessler H, Punsky I, Djaldetti M (1999) Effect of a garlic derivative (alliin) on peripheral blood cell immune responses. Int J Immunopharmacol 21: 589-597.

9 Wang BH, Zuzel KA, Rahman K, Billington D (1999) Treatment with 
aged garlic extract protects against bromobenzene toxicity to precision-cut rat liver slices. Toxicol 132: 215-225.

10 Chung LY (2006) The antioxidant properties of garlic compounds: Allyl cysteine, alliin, allicin, and allyl disulfide. J Med Food 9: 205-213.

11 Amagase $\mathrm{H}$ (2006) Clarifying the real bioactive constituents of garlic. J Nutr 136: 716S-725S.

12 Prette N, Monteiro AC, Garcia MV, Soares VE (2005) Patogenicidade de isolados de Beauveria bassiana para ovos, larvas e ninfas ingurgitadas de Rhipicephalus sanguineus. Ciênc Rural 35: 855-861.

13 Lei Y, Chen H, Sheen L, Lii C (2008) Diallydisulfide and diallyltrisulfide suppress oxidized LDL-induced vascular cell adhesion molecule and e-selectin expression through protein kinase A- and B-dependent signaling pathways. J Nutr 138: 996-1003.

14 Santhosha SG, Jamuna P, Prabhavathi SN (2013) Bioactive components of garlic and their physiological role in health maintainance: A review. Food Biosci 3: 59-74.

15 Lancaster JE, Shaw ML (1989) Y-Glutamyl peptides in the biosynthesis of S-alk(en)yl- L-cysteine sulfoxides (Flavour precursors) in Allium. Phytochem 28: 455-460.

16 Amagase $\mathrm{H}$, Petesch $\mathrm{BL}$, Matsuura $\mathrm{H}$, Kasuga S, Itakura $\mathrm{Y}(2001)$ Intake of garlic and its bioactive components. J Nutr 131: 955S-962S.

17 Fenwick GR, Hanley AB (1985) The Genus Allium Part 2. Critical Review Food Sci Nutr 22: 273-377.

18 Staba E, Staba J, Lash L, Staba JE (2001) A commentary on the effects of garlic extraction and formulation on product composition. J Nutr 131: 1118S- $1119 \mathrm{~S}$

19 Douaouya L, Bouzerna N (2016) Effect of garlic (Allium Sativum L) on biochemical parameters and histopathology of pancreas of alloxininduced diabetic in rats. Int J Pharm Pharm Sci 8: 202-206

20 Matos SL, Paula H, Pedrosa ML, Santos RC, Oliveira EL, et al. (2015) Dietary models for inducing hypercholesterolemia in rats. Brazilian Archives Biol Technol 48: 203-209.

21 El-Khedr MM (2017) Therapeutic benefits of garlic against alloxaninduced diabetic in rats. J Med Clin Res 5: 17445-17453

22 AOAC (2002) Official methods of analysis. Association of Analytical Chemists (17th edn) Washington DC, USA.

23 SAS (2002) SAS systems for windows 9.0, SAS INSTITUTE INC. CARY, NC, USA.

24 Harnafi H, Aziz M, Amrani S (2009) Sweet Basil (Ocimum basilicum L.) improves lipid metabolism in hypercholesterolemic rats E-SPEN, The European e-J. Clin Nutr Metabol 4: e181-e186.

25 Hartvigsen K, Binde CJ, Hansen LF, Rafia A, Juliano J, et al. (2007) A diet-induced hypercholesterolemic murine model to study atherogenesis without obesity and metabolic syndrome. Arterioscler Thromb Vasc Biol 27: 878-885.

26 Mohammadi A, Oshaghi EB (2014) Effect of garlic on lipid profile and expression of LXR alpa in Intestine and liver of hypercholesterolemic mice. J Diabetes Metabol Disorders 13: 20.

27 Bordia A, Bansal HC, Arora SK, Rathore AS, Ranawat RVS, et al. (1974) Effect of the essential oil (active principal) of garlic on serum cholesterol, plasma fibrinogen, whole blood coagulation time and fibrinolytic activity in alimentary lipaemia. J Assoc Phys Ind 22: 267-270.

28 Haque MR (1994) Effect of aqueous extract of garlic on serum and liver lipid profile of rats and its relevance to atherosclerosis (thesis). Dhaka: University of Dhaka, Bangladesh.

29 Zahid AM, Hussain ME, Fahim M (2005) Antiatherosclerotic effects of dietary supplementations of garlic and turmeric restoration of endothelial function in rats. Life Sci 77: 837-857.

30 Ide N, Lau BHS (1997) Garlic compounds protect vascular endothelial cells from oxidized low-density lipoprotein-induced injury. J Pharm Pharmacol 49: 908-911.

31 Yeh Y, Liu L (2001) Cholesterol effect of garlic extracts and organosulfur compounds: Human and animal studies. J Nutr 131: 989S-993S.

32 Sobenin IA, Andrianova IV, Fomchenkov IV, Gorchakova TV, Orekhov AN (2009) Time-released garlic powder tablets lower systolic and diastolic blood pressure in men with mild and moderate arterial hypertension. Hypertension Res 32: 433-437.

33 Getz GS, Reardon CA (2006) Diet and murine atherosclerosis. Arteriosclerosis, Thrombosis Vascular Biol 26: 242-249.

34 Jang MJ, Wang MH (2009) Effect of fermented soybean-derived chung koo kjang on diet-induced hyperlipidemia in Bio FIB hamsters. Food Biotechnol 23: 74-82.

35 Zacharias NT, Sebastian KL, Philip B, Augusti KT (1980) Hypoglycaemic and hypolipidaemic effects of garlic in sucrose fed rabbits. Ind J Physio Pharmac 24: 151-153.

36 Ebrahimi T, Behdad B, Abbsi MA, Rabati RG (2015) High dose of garlic extract significantly attenuated the ratio of serum LDL to HDL level in rats-fed with hypercholesterolemia diet. Diagnostic Pathol 10: 74.

37 Gonen A, Harat D, Rabinkov A, Miron T, Mirelman D, et al. (2005) The antiatherogenic effect of allicin: Possible mode of action. Pathobiol 72: 325-334.

38 Ali M, Al-Qattan KK, Al-Enezi F, Khanafer RM, Mustafa T (2000) Effect of allicin from garlic powder on serum lipids and blood pressure in rats fed with a high cholesterol diet. Prostaglandins, Leukotrienes and Essential Fatty Acids 62: 253-259.

39 Shouk R, Abdou A, Shetty K, Sarkar D, Eid AH (2014) Mechanisms underlying the anti-hypertensive effects of garlic bioactives. Nutr Res 34: 106-115. 